Supporting Information

\title{
A theoretical parameter-free analysis model for temperature programmed desorption (TPD) spectra
}

Jian $\mathrm{Xu} * 1,2$, Junyi Deng ${ }^{1}$

1. College of Materials Science and Engineering, Chongqing University, 400044, Chongqing, China.

2. Institute of Applied Physics, TU Wien, 1040 Vienna, Austria.

*corresponding author, email: jxu@cqu.edu.cn 

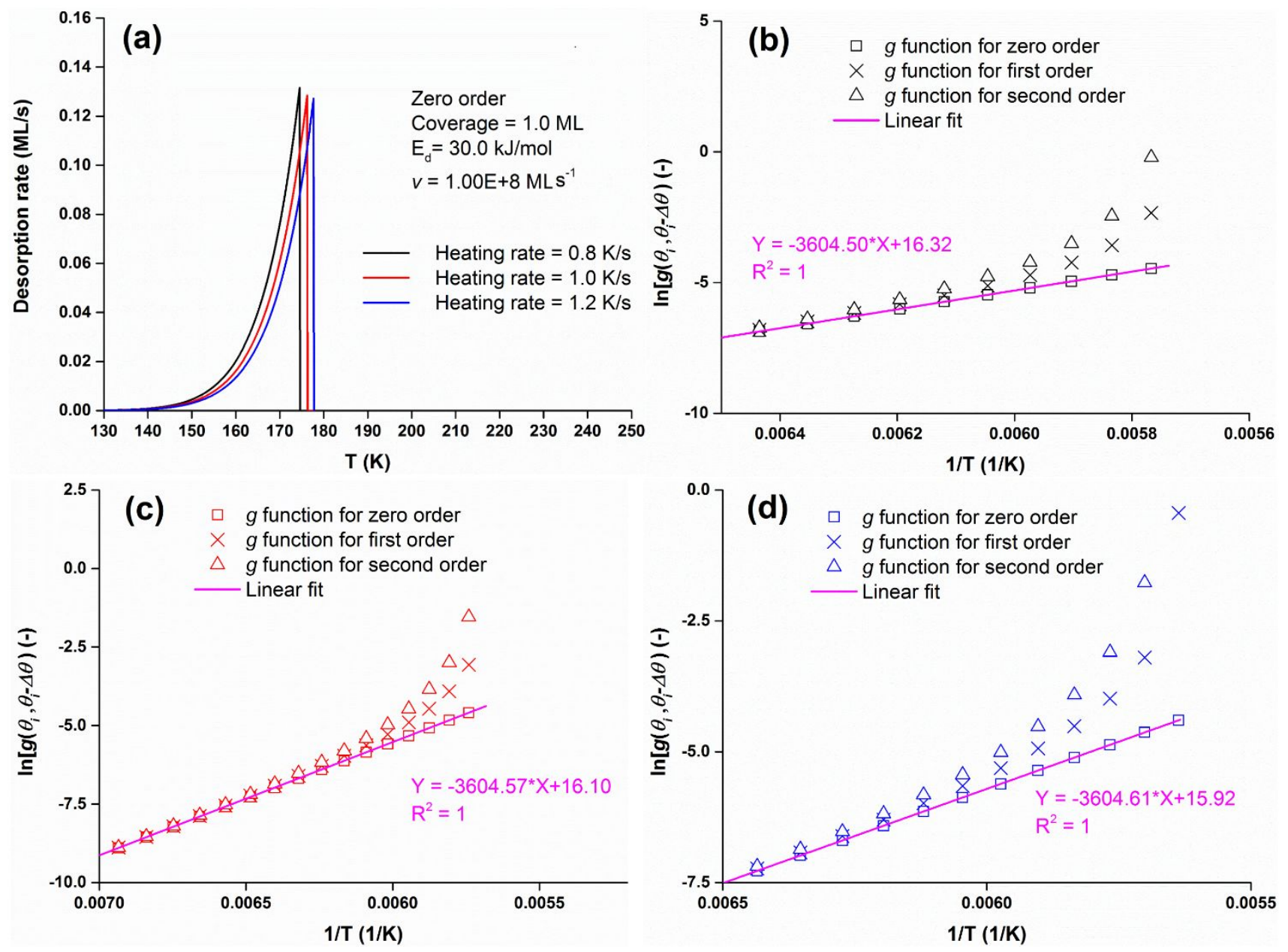

Figure S1 Model analysis on the three artificial mono-site spectra under different heating rates: (a) the input three mono-site spectra, and the model prediction results when the heating rate is (b) 0.8 $\mathrm{K} / \mathrm{s}$ (c) $1.0 \mathrm{~K} / \mathrm{s}$, (d) $1.2 \mathrm{~K} / \mathrm{s}$ 

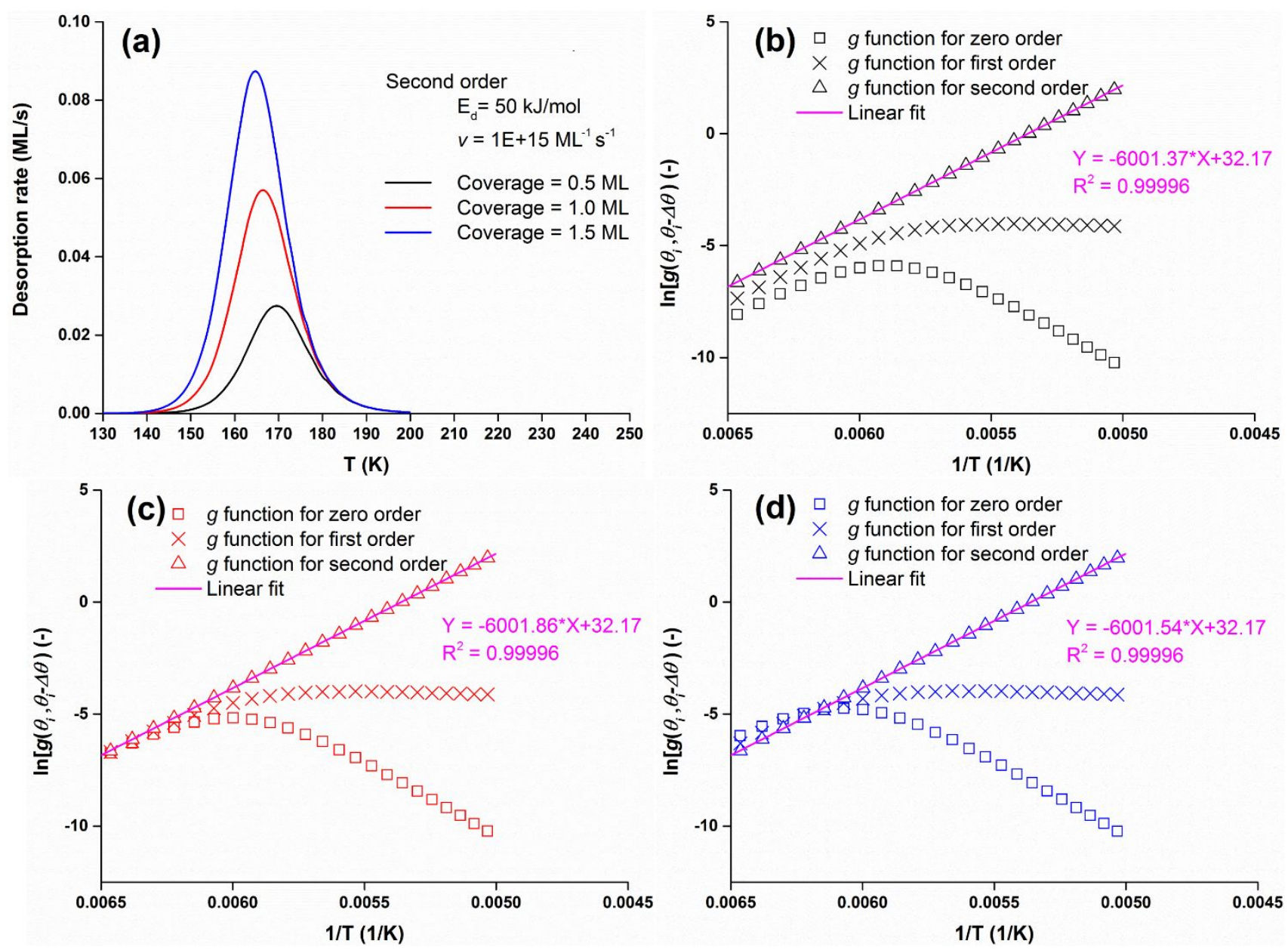

Figure S2 Model analysis on the three artificial mono-site spectra with different coverages: (a) the input three mono-site spectra, and the model prediction coverage-independent kinetic parameters when the initial surface coverage is (b) $0.5 \mathrm{ML}$, (c) $1.0 \mathrm{ML}$, (d) $1.5 \mathrm{ML}$ 

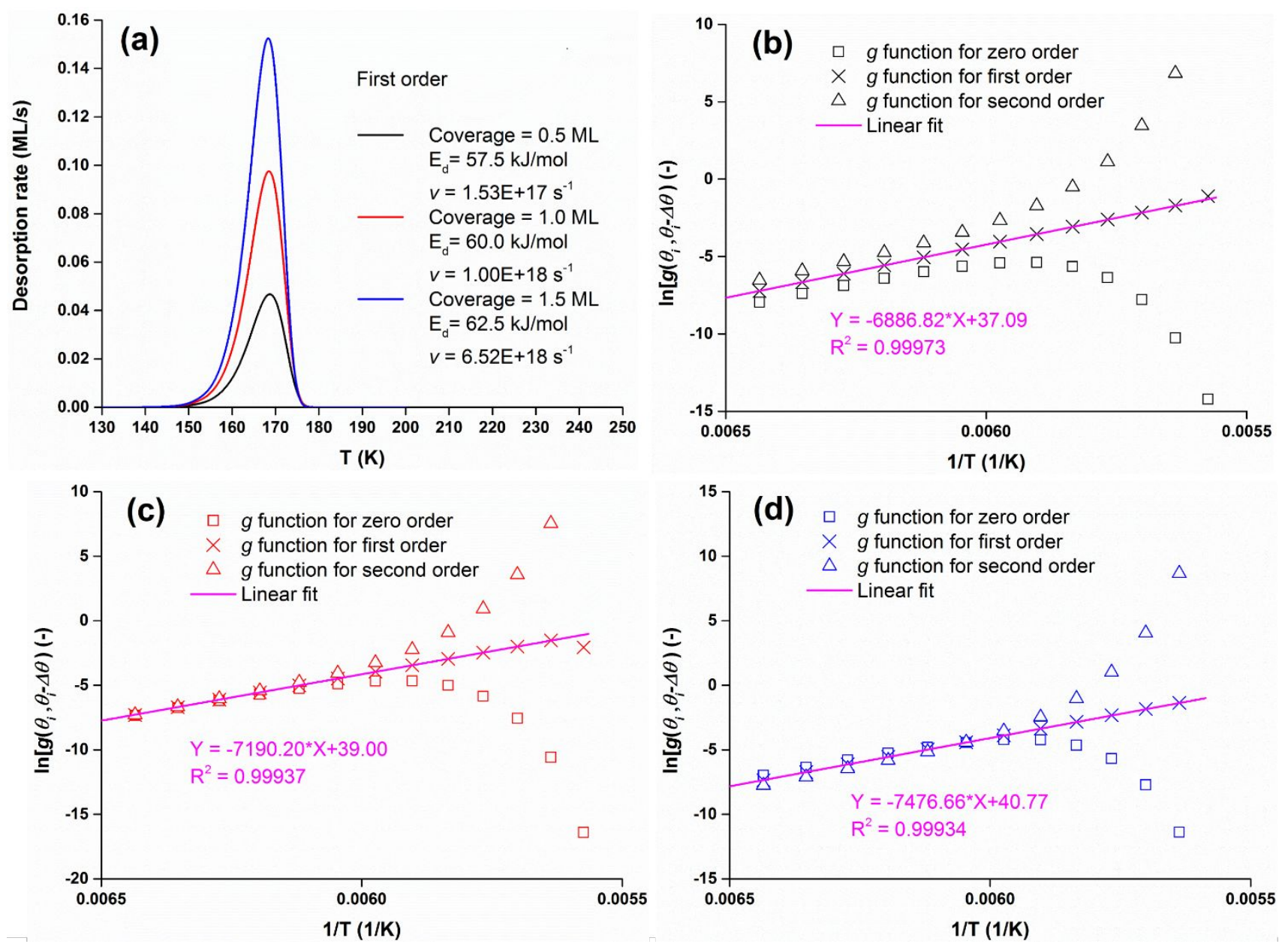

Figure S3 Model analysis on the three artificial mono-site spectra with coverage-dependent kinetic parameters: (a) the input three mono-site spectra, and the model prediction coveragedependent kinetic parameters when the initial coverage is (b) $0.5 \mathrm{ML}$, (c) $1.0 \mathrm{ML}$, (d) $1.5 \mathrm{ML}$ 


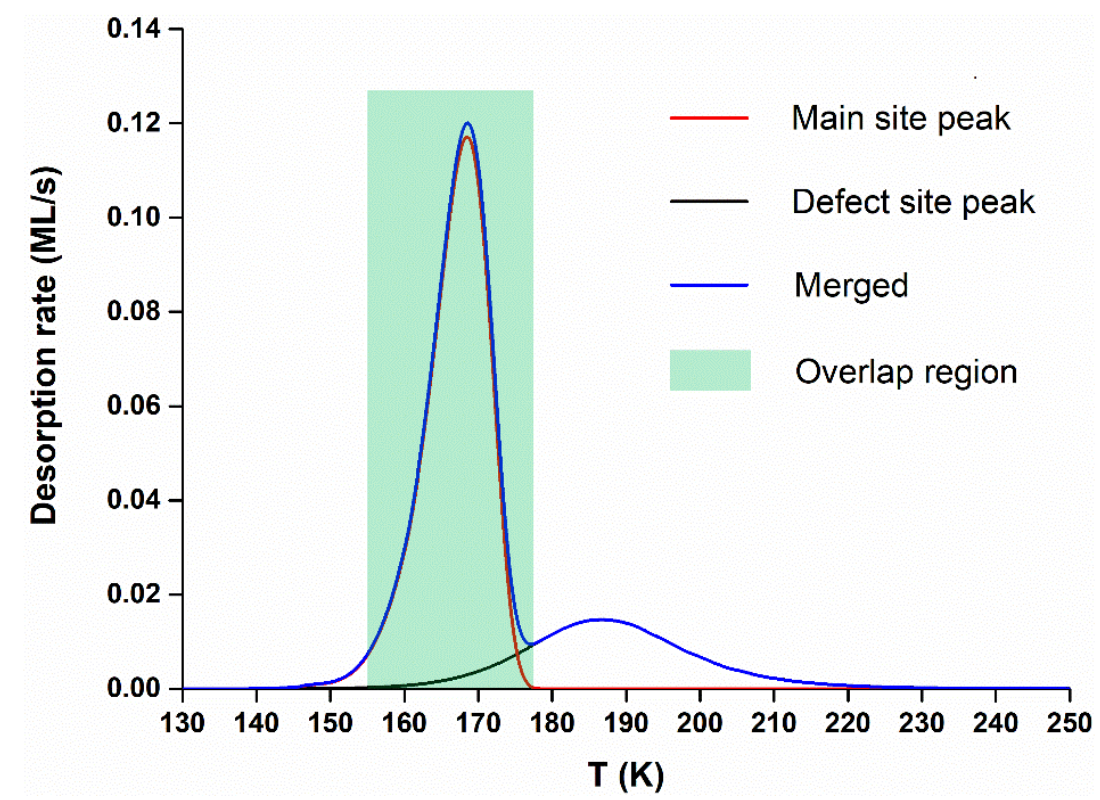

Figure S4 The overlap region in a two-site TPD spectrum 
Table S1 The model prediction results of a second order spectrum after 40 cycles

\begin{tabular}{cccccc}
\hline Cycle & $\begin{array}{c}\text { Starting } \\
\text { temperature } \\
(\mathrm{K})\end{array}$ & $\begin{array}{c}\text { Ending } \\
\text { temperature } \\
(\mathrm{K})\end{array}$ & $\begin{array}{c}E_{d} \\
(\mathrm{~kJ} / \mathrm{mol})\end{array}$ & $\begin{array}{c}\ln v \\
(-)\end{array}$ & $\begin{array}{c}\mathrm{R}^{2} \\
(-)\end{array}$ \\
\hline 40 & 180 & 189.9 & 39.8843 & 24.5515 & 1.0000 \\
41 & 182 & 191.9 & 40.1473 & 24.7227 & 0.9999 \\
42 & 184 & 193.9 & 40.3418 & 24.8465 & 0.9999 \\
43 & 186 & 195.9 & 40.3613 & 24.8576 & 0.9999 \\
44 & 188 & 197.9 & 39.9155 & 24.5775 & 0.9999 \\
45 & 190 & 199.9 & 39.5997 & 24.3829 & 0.9999 \\
46 & 192 & 201.9 & 39.914 & 24.5776 & 0.9998 \\
47 & 194 & 203.9 & 39.4951 & 24.3204 & 0.9994 \\
48 & 196 & 205.9 & 39.5883 & 24.3765 & 0.9991 \\
49 & 198 & 207.9 & 39.9217 & 24.5777 & 0.9989 \\
50 & 200 & 209.9 & 39.7278 & 24.4630 & 0.9988 \\
51 & 202 & 211.9 & 40.6845 & 25.0200 & 0.9992 \\
52 & 204 & 213.9 & 40.2718 & 24.7811 & 0.9992 \\
53 & 206 & 215.9 & 39.9989 & 24.6260 & 0.9995 \\
54 & 208 & 217.9 & 40.3185 & 24.8062 & 0.9995 \\
55 & 210 & 219.9 & 39.8188 & 24.5261 & 0.9994 \\
56 & 212 & 221.9 & 40.2463 & 24.7642 & 0.9992 \\
57 & 214 & 223.9 & 39.9039 & 24.5746 & 0.9990 \\
\hline
\end{tabular}


Table S2 The model prediction results of the $5 \%$ relative-noise affected TPD spectrum between 90 and 93 cycles

\begin{tabular}{cccccc}
\hline Cycle & $\begin{array}{c}\text { Starting } \\
\text { temperature } \\
(\mathrm{K})\end{array}$ & $\begin{array}{c}\text { Ending } \\
\text { temperature } \\
(\mathrm{K})\end{array}$ & $\begin{array}{c}E_{d} \\
(\mathrm{~kJ} / \mathrm{mol})\end{array}$ & $\begin{array}{c}\ln v \\
(-)\end{array}$ & $\begin{array}{c}\mathrm{R}^{2} \\
(-)\end{array}$ \\
\hline 90 & 190 & 239.9 & 48.6727 & 29.4819 & 0.4869 \\
91 & 191 & 240.9 & 49.8286 & 30.0553 & 0.4952 \\
92 & 192 & 241.9 & 50.3938 & 30.0411 & 0.4970 \\
93 & 193 & 242.9 & 50.5491 & 29.9468 & 0.4932 \\
\hline \multicolumn{7}{c}{} & & 50.2572 & 29.8813 & \\
\hline
\end{tabular}


Table S3 The model prediction results of the fixed-noise affected TPD spectrum between 81 and 85 cycles

\begin{tabular}{cccccc}
\hline Cycle & $\begin{array}{c}\text { Starting } \\
\text { temperature } \\
(\mathrm{K})\end{array}$ & $\begin{array}{c}\text { Ending } \\
\text { temperature } \\
(\mathrm{K})\end{array}$ & $\begin{array}{c}E_{d} \\
(\mathrm{~kJ} / \mathrm{mol})\end{array}$ & $\begin{array}{c}\ln v \\
(-)\end{array}$ & $\begin{array}{c}\mathrm{R}^{2} \\
(-)\end{array}$ \\
\hline 81 & 181 & 190.9 & 50.4338 & 30.1841 & 0.8491 \\
82 & 182 & 191.9 & 50.1293 & 29.9875 & 0.8558 \\
83 & 183 & 192.9 & 50.5016 & 30.2304 & 0.8736 \\
84 & 184 & 193.9 & 50.6498 & 30.3196 & 0.8813 \\
85 & 185 & 194.9 & 50.1707 & 30.0200 & 0.8718 \\
\hline \multicolumn{7}{c}{} \\
\hline
\end{tabular}


Table S4 The extracted data from TPD experiment of ammonia adsorbed at 293K (initial surface coverage $=100 \%$ ) by M.C. Abello et al. (Reuse with permission from ref. 32 Copyright 1995 Published by Elsevier B.V.)

\begin{tabular}{cc|cc|cc}
\hline $\mathrm{T}(\mathrm{K})$ & $\mathrm{A} . \mathrm{U} . / \mathrm{m}^{2}$ & $\mathrm{~T}(\mathrm{~K})$ & $\mathrm{A} . \mathrm{U} . / \mathrm{m}^{2}$ & $\mathrm{~T}(\mathrm{~K})$ & $\mathrm{A} . \mathrm{U} . / \mathrm{m}^{2}$ \\
\hline 295.3039 & 0.00134 & 367.1448 & 0.02702 & 512.691 & 0.01645 \\
297.7897 & 0.0018 & 372.1165 & 0.02712 & 514.3068 & 0.01569 \\
300.1513 & 0.00215 & 375.4724 & 0.02702 & 522.6344 & 0.01533 \\
302.6371 & 0.00282 & 379.574 & 0.02696 & 526.7361 & 0.01502 \\
308.4789 & 0.00302 & 383.6757 & 0.02686 & 533.3235 & 0.01487 \\
308.4789 & 0.00343 & 392.0033 & 0.0263 & 536.6794 & 0.01446 \\
310.9647 & 0.00385 & 397.7207 & 0.02615 & 543.2669 & 0.01364 \\
313.4506 & 0.0042 & 403.5624 & 0.02553 & 560.6678 & 0.01318 \\
315.0664 & 0.00487 & 410.1499 & 0.02522 & 575.4586 & 0.0122 \\
317.5522 & 0.00641 & 417.6075 & 0.02481 & 591.2437 & 0.0122 \\
320.9081 & 0.00702 & 422.5792 & 0.02307 & 601.1871 & 0.0121 \\
322.5239 & 0.00846 & 424.195 & 0.02261 & 611.0062 & 0.01189 \\
325.0097 & 0.00923 & 430.0367 & 0.02148 & 621.8196 & 0.01148 \\
328.3656 & 0.01138 & 435.7541 & 0.02066 & 639.9663 & 0.01113 \\
329.9814 & 0.01231 & 449.7992 & 0.02035 & 654.8814 & 0.01015 \\
332.4673 & 0.01379 & 439.11 & 0.01984 & 668.9264 & 0.01015 \\
336.5689 & 0.0162 & 443.2117 & 0.01938 & 675.5139 & 0.00943 \\
339.0548 & 0.01718 & 451.5393 & 0.01871 & 686.3273 & 0.00933 \\
341.5406 & 0.01896 & 462.2284 & 0.01825 & 722.6206 & 0.00774 \\
344.8965 & 0.02041 & 465.5843 & 0.01784 & 735.9199 & 0.00708 \\
348.1281 & 0.02246 & 474.6576 & 0.01794 & 760.6541 & 0.00584 \\
353.0998 & 0.02327 & 481.2451 & 0.01722 & 774.6991 & 0.00507 \\
354.7156 & 0.02471 & 486.2168 & 0.01656 & 793.7158 & 0.00513 \\
358.9415 & 0.02589 & 496.1602 & 0.01671 & 807.0151 & 0.00513 \\
363.0432 & 0.0264 & 504.3635 & 0.01651 & 818.5742 & 0.00513 \\
\hline
\end{tabular}

